\section{MR Imaging of the Midfoot and Forefoot}

MR imaging is the diagnostic modality of choice for the evaluation of traumatic, inflammatory, and neoplastic processes affecting the midfoot and forefoot soft tissue structures, including muscles, ligaments and tendons. MR imaging is also valuable in the diagnosis of occult bony trauma, stress reaction or fractures, and osteomyelitis. Exquisite contrast resolution, noninvasiveness, and multiplanar capabilities are unique features that make MR imaging a powerful diagnostic technique. The authors employ a Basic Protocol using $T_{1}$-weighted, fast spin echo proton density with and without frequency-selective fat saturation, as well as fast spin echo $T_{2}$-weighted sequences and short-tau inversion recovery (STIR) imaging. Additional imaging following intravenous gadolinium administration is indicated when osteomyelitis and neoplastic processes are suspected. Post-contrast fat-suppressed $T_{1}$-weighted sequences are necessary if intravenous or intraarticular gadolinium is utilized.

\section{EXAMINATION OF THE MIDFOOT AND FOREFOOT}

Most reports on MR imaging of the foot available in the literature are based on studies performed on high-strength (1.5 T) magnets (Zlatkin, 1999). The following sequences described herein are based on the author's experience with a GE $1.5 \mathrm{~T}$ scanner, but are expected to be applicable to other equipment manufacturers. The entire protocol takes $\sim 20 \mathrm{~min}$ to scan the patient and roughly $30 \mathrm{~min}$ to perform when patient setup time is included. If post-gadolinium imaging is obtained, 8 additional minutes are required.

Table A25.2.1 lists the six sequences necessary to perform the Basic Protocol, along with one optional sequence. Stepwise instructions for performing the imaging protocol are provided. This protocol should be easily acquired on most clinical systems.

Table A25.2.2 lists the hardware required to perform the procedure, along with the appropriate parameters. The available gradient strength will depend on the scanner, and the echo times given below may be varied accordingly (the smaller the gradient strength, the longer the echo time for a particular scan).

When using mid/low field $(<1.5 \mathrm{~T})$ scanners, adjustments to the imaging protocols need to be made in order to compensate for the lower signal-to-noise ratio (SNR) related to lower-field strength and gradient strength. In general, MRI examinations obtained in mid/low-field machines require longer acquisition times and a larger field of view. Signal

Table A25.2.1 Midfoot and Forefoot Protocol

\begin{tabular}{ll}
\hline Type of weighting and sequence & Imaging plane \\
\hline $\begin{array}{l}\text { Gradient echo three-plane positioning } \\
\text { scout }\end{array}$ & $\begin{array}{l}\text { Sagittal, coronal, and } \\
\text { transverse }\end{array}$ \\
Proton density-weighted fast spin echo & Oblique coronal \\
$T_{2}$-weighted fast spin echo & Oblique coronal \\
Proton density-weighted fat suppressed & Oblique transverse \\
fast spin echo & \\
$T_{1}$-weighted spin echo & Oblique sagittal \\
Turbo STIR & Oblique sagittal \\
Optional sequence & \\
$T_{1}$-weighted post-gadolinium & Oblique coronal(s) \\
fat-suppressed gradient echo & \\
\hline
\end{tabular}

Contributed by Jenny Bencardino and Zehava S. Rosenberg

Current Protocols in Magnetic Resonance Imaging (2008) A25.2.1-A25.2.15

Copyright $(\underset{2}{ } 2008$ by John Wiley \& Sons, Inc.
UNIT A25.2

BASIC

PROTOCOL

Ankle and Foot

A25.2.1

Supplement 15 


\begin{tabular}{ll}
\hline Coil type & Quadrature extremity coil \\
Gradient coil strength & $25 \mathrm{mT} / \mathrm{m}$ (or whatever the system \\
& permits) \\
Cardiac gating & No \\
Respiratory gating & No \\
Peripheral monitoring & For safety only \\
Respirator/oxygen & If required by the patient \\
Motion cushions & Useful \\
\hline
\end{tabular}

abnormalities related to increased water content in tissue must be evaluated using STIR sequences, since frequency-selective fat saturation cannot be obtained when using these systems.

NOTE: Be sure that technologists and nurses have immediate access to any emergency equipment that may be relevant to a given study, or that may be needed for a particular patient, such as crash carts or oxygen.

\section{Materials}

Extravascular contrast agent (Omniscan, Magnevist, Prohance)

Normal saline $(0.9 \% \mathrm{NaCl})$, sterile

\section{Set up patient and equipment}

1. Interview the patient to assess for contraindications such as cardiac pacemakers or other implants containing ferromagnetic materials. Also, determine if the patient will need sedation medication requiring the use of monitoring equipment.

A screening form should be filled and signed by each patient or legal guardian prior to scanning in a magnetic resonance system. Generally, standard screening forms (see APPENDIX 1) are used for all patients scanned in a magnetic resonance system.

The presence of ferromagnetic material in a patient may represent a health hazard to the patient while he or she is inside the magnet and/or deteriorate image quality. The safety of such ferromagnetic materials is discussed in Shellock (2007).

Patients with previous metal exposure to the eyes should have radiographs of the orbits in order to exclude metallic foreign bodies that have not been removed, prior to placing them in the magnetic field.

A friend or family member, who can sit in the room during the scan and comfort the patient as needed, may accompany patients into the magnet room. This companion must be screened as well to ensure the absence of loose metal objects on the body or clothing.

2. If the procedure is a research protocol, have the patient sign any necessary consent form.

3. Have the patient remove jewelry and change into a gown to eliminate any metal that might be found in clothing. All personal belongings should be secured properly during the examination.

4. Have the patient wash off any mascara and other makeup to avoid local tissue heating and image artifacts.

5. Inform the patient about what will occur during the procedure, what he or she will experience while in the magnet, and how to behave.

MR Imaging of the Midfoot and Forefoot

\section{A25.2.2}


a. If earplugs, earphones, and/or headphones are used to protect the ears from the loud sounds produced by the gradients, the patient will be asked to wear these, but will be able to communicate with you at any time during the imaging.

b. The patient will be given a safety squeeze-bulb or similar equipment to request assistance at any time (demonstrate how this works).

c. For good results, the patient should not talk, and should avoid or minimize other movements, during each scan-i.e., as long as the banging sounds continue. Between scans, talking is allowed in most cases, but should be avoided when comparative positional studies are being performed; the patient will be informed when this is the case.

d. Nevertheless, the patient may call out at any time if he or she feels it necessary.

6. Ask the patient to remain perfectly still during imaging acquisition and also explain the need not to move between imaging sequences.

7. Have the patient lie on the table, with the feet toward the bore of the magnet. For imaging of the midfoot and forefoot, we recommend that the patient lie supine with the ankle in neutral position. Either before or right after the patient lies down, set up any triggering devices or other monitoring equipment that is to be used.

The foot in neutral position has an inherent $10^{\circ}$ to $20^{\circ}$ of plantar flexion and $10^{\circ}$ to $30^{\circ}$ degrees of external rotation. Neutral positioning of the foot is often well tolerated by most patients.

8. Center the foot to be examined in the extremity coil and use the laser light to position the ankle joint level.

Mechanical stabilization of the foot is paramount for good image quality. Immobilization should be achieved by using foam sponges between the foot and the coil and Velcro straps on the leg outside the coil.

9. Advance the patient table to isocenter.

Once this step has been performed, so long as the patient does not move on the table, the table itself can be moved and then placed in the same position as before without jeopardizing the positioning of one scan relative to another.

10. If the patient is unable to hold still, provide an appropriate sedative if indicated.

\section{Sequence 1: Three-plane positioning scout}

11. To verify the patient's position, run the scout scan to ensure correct location of the foot in three dimensions using the imaging parameters provided in Table A25.2.3. This sequence will be used to set up coverage of the area of interest. Most MR systems can be programmed to acquire the scout images automatically after coil tuning or after the patient has been placed in the isocenter.

\section{Sequence 2: Oblique coronal FSE proton-density weighted}

12. Using the images generated in sequence 1, prescribe oblique coronal FSE (fast spin echo) proton density-weighted images to provide complete coverage of the midfoot and forefoot (Fig. A25.2.1). The sagittal scout is the best plane for planning this oblique coronal sequence, which is prescribed perpendicular to the metatarsals and plantar surface of the foot. Check the coverage of the coronal scan on the transverse scout image. Use this oblique coronal sequence to position subsequent scans accurately. Run sequence 2 according to Table A25.2.4. 
Patient position

Scan type

Imaging plane (orientation)

Central slice or volume center

Echo time $\left(T_{\mathrm{E}}\right)$

Repeat time $\left(T_{\mathrm{R}}\right)$

Flip angle (FA)

Fields of view $\left(\mathrm{FOV}_{x}, \mathrm{FOV}_{y}\right)$

Resolution $(\Delta x, \Delta y)$

Number of data points collected $\left(N_{x}, N_{y}\right)$

Slice thickness $(\Delta z)$

Number of slices

Slice gap

Number of acquisitions $\left(N_{\text {acq }}\right)$

Scan time
Supine

Gradient echo

Three planes

Magnet isocenter

$4.2 \mathrm{msec}$

$12.1 \mathrm{msec}$

$30^{\circ}$

$240 \mathrm{~mm}, 240 \mathrm{~mm}$

$1.88 \mathrm{~mm}, 1.88 \mathrm{~mm}$

128,128

$4 \mathrm{~mm}$

9 (3 in each of 3 cardinal planes)

$1 \mathrm{~mm}$

1

$1 \mathrm{~min}, 5 \mathrm{sec}$
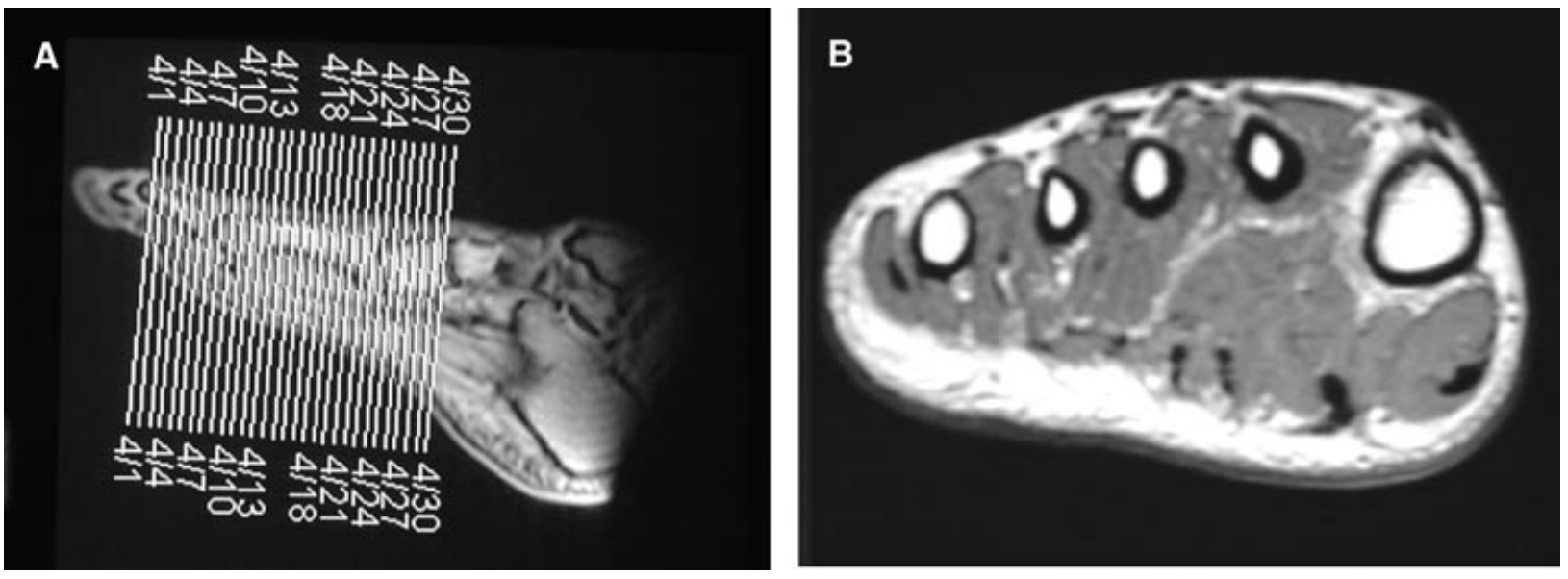

Figure A25.2.1 Oblique coronal imaging plane. (A) Using the sagittal localizer, oblique coronal FSE proton densityweighted images can be prescribed perpendicular to the metatarsals covering from the Chopart joint to the toes. The foot is in neutral position. (B) An oblique coronal FSE proton density-weighted image through the metatarsal shafts. Note the exquisite anatomic detail.

MR Imaging of the Midfoot and Forefoot

A25.2.4

\section{Sequence 3: Oblique coronal fat-suppressed FSE $T_{2}$ weighted}

13. Set parameters for the coronal FSE $T_{2}$-weighted sequence as indicated in Table A25.2.5. Start the scan.

This scan is positioned as in sequence 2. The coverage should include the midfoot and forefoot structures as well. This sequence will provide demonstration of alterations in water content characteristic of most pathologic conditions in the foot and ankle. Fatsuppressed imaging may be utilized to highlight the contrast differences between the low-signal tendinous and ligamentous structures and the abnormal free water.

\section{Sequence 4: Oblique transverse fat-suppressed FSE proton-density weighted}

14. Set parameters for the oblique transverse fat-suppressed FSE proton densityweighted sequence as indicated in Table A25.2.6. Using the oblique coronal images generated in sequence 2, prescribe oblique transverse fat-suppressed FSE proton density-weighted images parallel to the metatarsals and to the plantar surface of the 
Patient position

Scan type

Imaging plane (orientation)

Variable bandwidth

Central slice or volume center

Echo time $\left(T_{\mathrm{E}}\right)$

Receiver bandwidth (RBW)

Echo train length (ETL)

Repeat time $\left(T_{\mathrm{R}}\right)$

Flip angle (FA)

Fields of view $\left(\mathrm{FOV}_{x}, \mathrm{FOV}_{y}\right)$

Resolution $(\Delta x, \Delta y)$

Number of data points collected $\left(N_{x}, N_{y}\right)$

Slice thickness $(\Delta z)$

Number of slices

Slice gap

Number of acquisitions $\left(N_{\text {acq }}\right)$

Read direction

Scan time

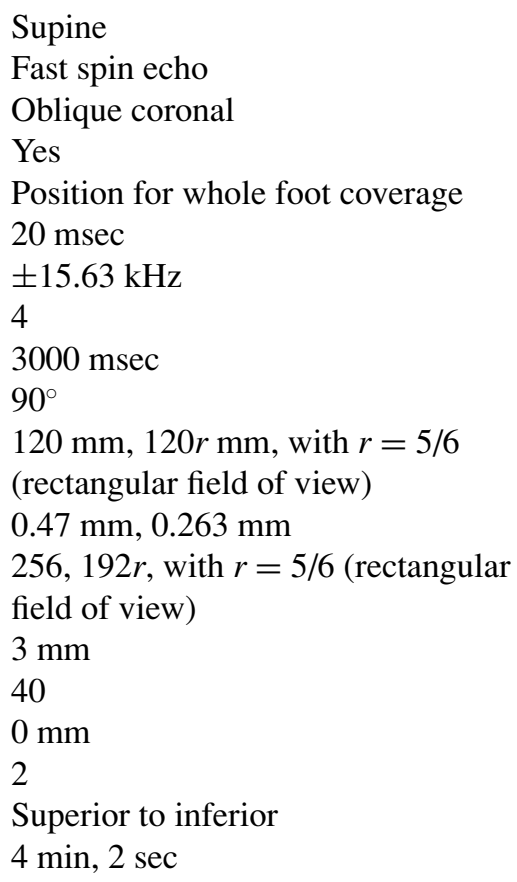

Table A25.2.5 Parameters for Coronal FSE $T_{2}$ Weighted

\begin{tabular}{ll}
\hline Patient position & Supine \\
Scan type & Fast spin echo \\
Imaging plane (orientation) & Oblique coronal \\
Variable bandwidth & Yes \\
Central slice or volume center & Position for whole foot coverage \\
Echo time $\left(T_{\mathrm{E}}\right)$ & $80 \mathrm{msec}$ \\
Receiver bandwidth $(\mathrm{RBW})$ & $\pm 15.63 \mathrm{kHz}$ \\
Echo train length $(\mathrm{ETL})$ & 16 \\
Repeat time $\left(T_{\mathrm{R}}\right)$ & $3600 \mathrm{msec}$ \\
Flip angle $(\mathrm{FA})$ & $90^{\circ}$ \\
Fields of view (FOV, FOV & \\
& $120 \mathrm{~mm}, 120 \mathrm{r}$ mm, with $r=5 / 6$ \\
Resolution $(\Delta x, \Delta y)$ & $(\mathrm{rectangular}$ field of view) \\
Number of data points collected $\left(N_{x}, N_{y}\right)$ & $0.47 \mathrm{~mm}, 0.263 \mathrm{~mm}$ \\
& $256,192 r$, with $r=5 / 6$ (rectangular \\
Slice thickness $(\Delta z)$ & field of view) \\
Number of slices & $3 \mathrm{~mm}$ \\
Slice gap & 40 \\
Number of acquisitions $\left(N_{\text {acq }}\right)$ & $1 \mathrm{~mm}$ \\
Read direction & 2 \\
No phase wrap $(\mathrm{NPW})$ & Superior to inferior \\
Fat suppression & Yes \\
Scan time & Yes \\
\hline
\end{tabular}

foot (Fig. A25.2.2). Check the coverage of the transverse scan on the sagittal scout. Perform system shim as recommended by manufacturer, as this is a fat-saturation sequence. Begin the scan.

\section{Sequence 5: Oblique sagittal $T_{1}$-weighted spin-echo}

15. Set parameters for the $T_{1}$-weighted spin echo sagittal sequence as given in Table A25.2.7. Using the oblique transverse images generated in sequence 4, prescribe 
Table A25.2.6 Parameters for Transverse Fat-Suppressed FSE Proton-Density Weighted

Patient position

Scan type

Imaging plane (orientation)

Variable bandwidth

Central slice or volume center

Echo time $\left(T_{\mathrm{E}}\right)$

Receiver bandwidth (RBW)

Echo train length (ETL)

Repeat time $\left(T_{\mathrm{R}}\right)$

Flip angle (FA)

Fields of view $\left(\mathrm{FOV}_{x}, \mathrm{FOV}_{y}\right)$

Resolution $(\Delta x, \Delta y)$

Number of data points collected $\left(N_{x}, N_{y}\right)$

Slice thickness $(\Delta z)$

Number of slices

Slice gap

Number of acquisitions $\left(N_{\text {acq }}\right)$

Read direction

Tailored RF

Fat suppression

Scan time
Supine

Fast spin echo

Oblique transverse

Yes

Position for whole foot coverage

$29 \mathrm{msec}$

$\pm 15.63 \mathrm{kHz}$

6

$2500 \mathrm{msec}$

$90^{\circ}$

$140 \mathrm{~mm}, 140 r \mathrm{~mm}$, with $r=5 / 7$

(rectangular field of view)

$0.55 \mathrm{~mm}, 0.273 \mathrm{~mm}$

256, 192r, with $r=5 / 7$ (rectangular field of view)

$2.5 \mathrm{~mm}$

20

0

2

Anterior to posterior

Yes

Yes

$2 \mathrm{~min}, 10 \mathrm{sec}$
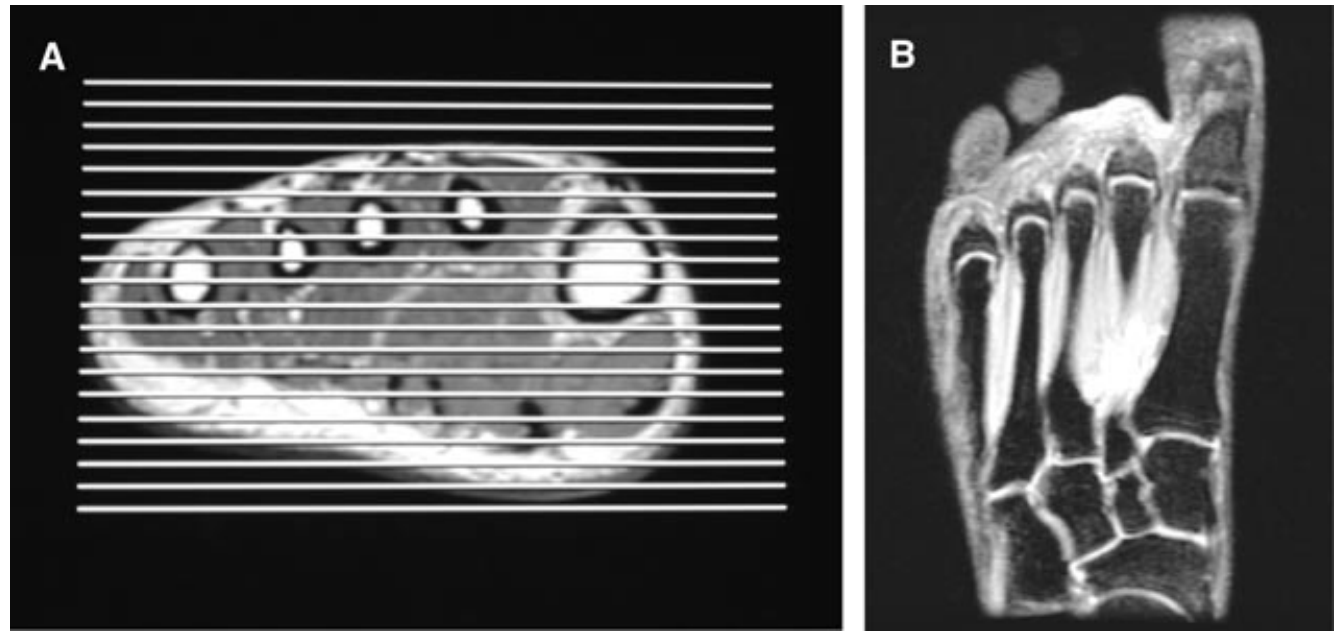

Figure A25.2.2 Oblique transverse imaging plane. (A) Based on oblique coronal FSE proton densityweighted image, the oblique transverse-transverse slices are prescribed parallel to the metatarsals. (B) Oblique axial fat-suppressed FSE proton-density image through the midfoot and forefoot.

MR Imaging of the Midfoot and Forefoot

A25.2.6 
Table A25.2.7 Parameters for Sagittal $T_{1}$-Weighted Spin-Echo

Patient position

Scan type

Imaging plane (orientation)

Variable bandwidth

Central slice or volume center

Echo time $\left(T_{\mathrm{E}}\right)$

Receiver bandwidth (RBW)

Repeat time $\left(T_{\mathrm{R}}\right)$

Flip angle (FA)

Fields of view $\left(\mathrm{FOV}_{x}, \mathrm{FOV}_{y}\right)$

Resolution $(\Delta x, \Delta y)$

Number of data points collected $\left(N_{x}, N_{y}\right)$

Slice thickness $(\Delta z)$

Number of slices

Slice gap

Number of acquisitions $\left(N_{\text {acq }}\right)$

Read direction

Extended dynamic range (EDR)

Scan time
Supine

Spin echo

Oblique sagittal

Yes

Position for whole foot coverage

Minimum (e.g., $15 \mathrm{msec}$ )

$\pm 15.63 \mathrm{kHz}$

$500 \mathrm{msec}$

$90^{\circ}$

$150 \mathrm{~mm}, 150 \mathrm{~mm}$

$0.59 \mathrm{~mm}, 0.78 \mathrm{~mm}$

256, 192

$3 \mathrm{~mm}$

20

$1 \mathrm{~mm}$

2

Superior to inferior

Yes

$3 \mathrm{~min}, 20 \mathrm{sec}$
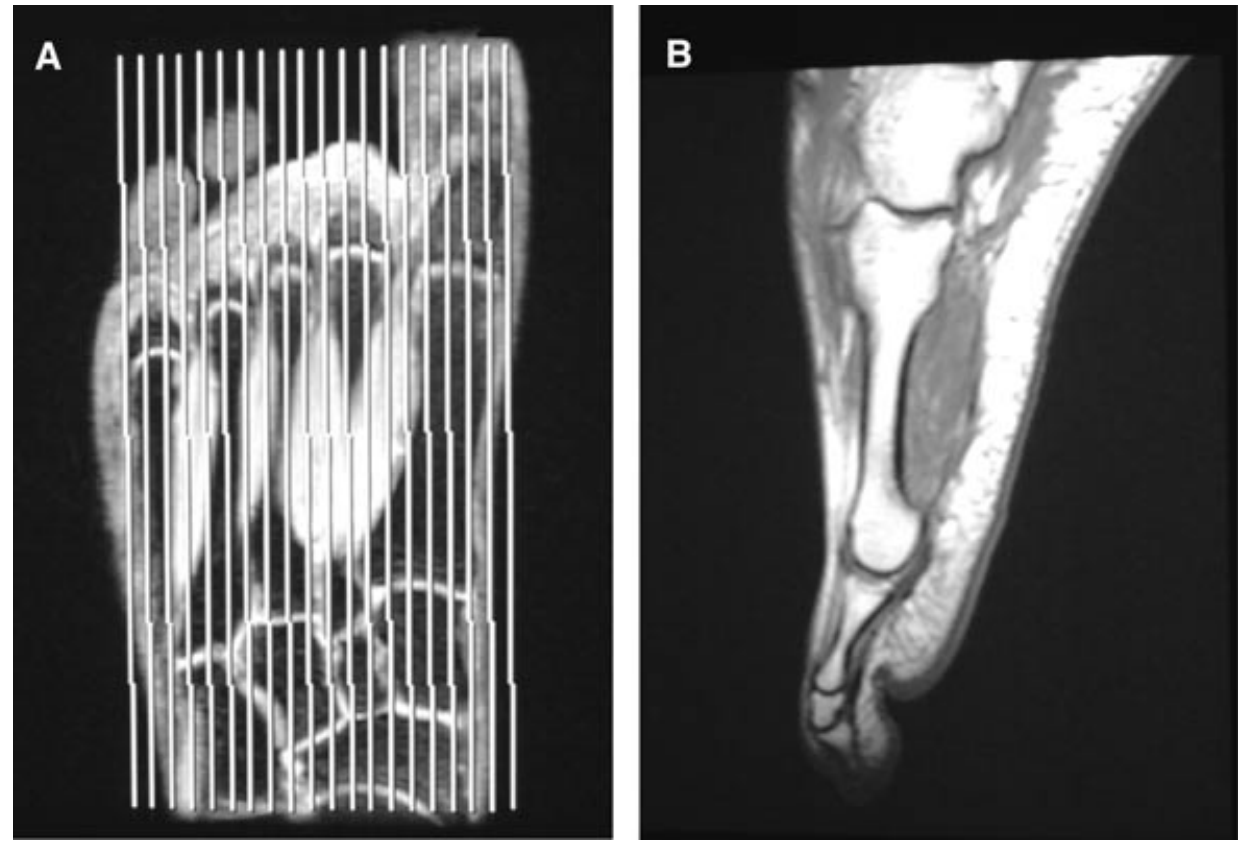

Figure A25.2.3 Oblique sagittal imaging plane. (A) Based on transverse fat-suppressed FSE proton-density weighted image, the sagittal plane should be aligned parallel to the metatarsal shafts. (B) A sagittal $T_{1}$-weighted spin-echo image along the second metatarsal shaft.

oblique sagittal images to provide complete coverage of the midfoot and forefoot. Start the scan.

Some angulation is needed to provide true sagittal anatomic imaging. The imaging plane should be parallel to the shafts of the metatarsals (Fig. A25.2.3).

This plane is particularly useful for evaluating fractures of the metatarsals, malalignment of the midfoot and forefoot articulations, and osteochondral injuries of the navicular and metatarsal heads. 


\section{Sequence 6: Oblique sagittal turbo STIR}

16. Set parameters for the sagittal turbo STIR sequence as indicated in Table A25.2.8. Use the fat-suppressed FSE proton density-weighted oblique transverse images to position the sagittal scans. Align the imaging plane parallel to the second through fifth metatarsal shafts (Fig. A25.2.3). When clinical concern is centered at the great toe, prescribe the imaging plane along the main axis of the first metatarsal (Rubin et al., 1996).

\section{Sequence 7: Post-gadolinium fat-suppressed $T_{1}$-weighted spin echo (optional)}

When osteomyelitis or neoplastic processes are suspected, additional imaging following intravenous administration of gadolinium is recommended.

17. Pull the patient out of the magnet.

18. Establish an intravenous line from which the contrast agent can be injected, and attach this line securely to the patient so that movement into or out of the magnet will not pull at the patient's arm.

It is preferable to insert the line prior to imaging and to leave the patient in the magnet, with no intervening motion, between the scans run before contrast agent injection and those run after injection.

19. Repeat steps 8 and 9.

20. If the patient moved, run sequence 1 again to ensure the position of the patient.

21. Leave the patient in the magnet, inject the contrast agent, and flush the line with $10 \mathrm{ml}$ saline.

A dose of $0.1 \mathrm{mmol} / \mathrm{kg}$ of contrast agent is usually given.

22. Run sequence 7 according to Table A25.2.9.

Table A25.2.8 Parameters for Sagittal Turbo STIR

\begin{tabular}{ll}
\hline Patient position & Supine \\
Scan type & STIR \\
Imaging plane (orientation) & Oblique sagittal \\
Variable bandwidth & Yes \\
Central slice or volume center & Position for whole foot coverage \\
Echo time $\left(T_{\mathrm{E}}\right)$ & $21 \mathrm{msec}$ \\
Receiver bandwidth $(\mathrm{RBW})$ & $\pm 31.25 \mathrm{kHz}$ \\
Echo train length $(\mathrm{ETL})$ & 6 \\
Repeat time $\left(T_{\mathrm{R}}\right)$ & $4200 \mathrm{msec}$ \\
Inversion time $\left(T_{\mathrm{I}}\right)$ & $130 \mathrm{msec}$ \\
Flip angle $(\mathrm{FA})$ & $180^{\circ}$ \\
Fields of view $\left(\mathrm{FOV}, \mathrm{F}_{x}, \mathrm{FOV}\right.$ & \\
Resolution $(\Delta x, \Delta y)$ & $150 \mathrm{~mm}, 150 \mathrm{~mm}$ \\
Number of data points collected $\left(N_{x}, N_{y}\right)$ & $0.59 \mathrm{~mm}, 0.78 \mathrm{~mm}$ \\
Slice thickness $(\Delta z)$ & 256,192 \\
Number of slices & $3 \mathrm{~mm}$ \\
Slice gap & 20 \\
Number of acquisitions $\left(N_{\text {acq }}\right)$ & $1 \mathrm{~mm}$ \\
Read direction & 2 \\
Scan time & Superior to inferior \\
\hline
\end{tabular}

MR Imaging of the Midfoot and Forefoot

A25.2.8 
Table A25.2.9 Parameters for Post-Gadolinium Fat-Suppressed $T_{1}$-Weighted Spin Echo

\begin{tabular}{ll}
\hline Patient position & Supine \\
Scan type & Spin echo \\
Imaging plane (orientation) & Oblique coronal \\
Central slice or volume center & Position for whole foot coverage \\
Echo time $\left(T_{\mathrm{E}}\right)$ & Minimum (e.g., $15 \mathrm{msec})$ \\
Repeat time $\left(T_{\mathrm{R}}\right)$ & $800 \mathrm{msec}$ \\
Flip angle $(\mathrm{FA})$ & $90^{\circ}$ \\
Fields of view $\left(\mathrm{FOV}_{x}, \mathrm{FOV}_{y}\right)$ & $140 \mathrm{~mm}, 140 \mathrm{rmm}$ \\
Resolution $(\Delta x, \Delta y)$ & $0.55 \mathrm{~mm}, 0.273 \mathrm{~mm}$ \\
Number of data points collected $\left(N_{x}, N_{y}\right)$ & $256,192 r$ \\
Slice thickness $(\Delta z)$ & $3 \mathrm{~mm}$ \\
Number of slices & 20 \\
Slice gap & $0 \mathrm{~mm}$ \\
Number of acquisitions $\left(N_{\mathrm{acq}}\right)$ & 2 \\
Read direction & Cranial-caudal \\
Fat suppression & Yes \\
Scan time & $3 \mathrm{~min}, 54 \mathrm{sec}$ \\
\hline
\end{tabular}

\section{COMMENTARY}

\section{Background Information}

\section{Forefoot pain}

Forefoot pain is a common clinical complaint that can result in significant limitation of function. Patients with Morton's neuroma classically experience sharp pain, burning sensation, and paresthesias during weight bearing in the region of the intermetatarsal spaces. The pain is relieved by rest and shoe removal. On physical examination, a mass can be palpated in a third of the cases. This is often accompanied by a characteristic click or Mulder's sign. In some cases, the diagnosis may be equivocal and other causes of intermetatarsalgia may be clinically entertained. The differential diagnosis includes intermetatarsal bursitis, true neuroma, inflammatory arthritis, pigmented villonodular synovitis, osteomyelitis, foreign-body granuloma, stress fracture, Freiberg's infraction, and metatarsophalangeal joint dislocation. In these instances, MR imaging has proven to be valuable in establishing the underlying cause of intermetatarsal pain (Bencardino et al., 2000; Ashman et al., 2001). Additionally, if surgical management is considered, MR examination can confirm the diagnosis and determine the number and exact location of the neuromas. Furthermore, in patients with persistent pain following surgical excision of Morton's neuroma, MRI imaging can help to delineate the presence of a recurrent neuroma in the surgical bed or a separate neuroma in a different intermetatarsal space. Prone imaging has also been advocated (Weishaupt et al., 2003).

\section{Soft tissue masses}

Ganglions are unilocular or multilocular cystic lesions filled with mucoid material and surrounded by a fibrous capsule. Although most ganglions communicate with an adjacent joint or tendon sheath, some of them may be completely separate. The foot is the second most common site of occurrence after the hand and wrist. They are often located in the dorsal aspect of the foot or in the region of the sinus tarsi where they can compress the posterior tibial nerve resulting in tarsal tunnel syndrome (Weishaupt et al., 2001). Clinically, ganglions present as focal masses producing dull pain or limitation of motion. Treatment consists of excision of the ganglion cyst in its entirety, to prevent local recurrence. MRI without and with contrast agent is indicated in order to exclude the presence of enhancing internal nodular components that if present can suggest a neoplastic process such as a myxomatous tumor or synovial sarcoma.

Pigmented villonodular synovitis is a condition characterized by inflammatory, nonneoplastic proliferation of the synovium associated with hemosiderin deposition. Pigmented villonodular synovitis (PVNS) typically affects adults at ages between 20 and 50 years. It can be present in any joint, tendon sheath, or bursa, but it is seen more frequently in the knee, hip, ankle, and elbow. When it 
originates in the tendon sheaths, the term "giant cell tumor of the tendon sheaths" is often used. In the foot, giant cell tumor of the tendon sheaths involves predominantly the periarticular tendon sheath complex of small joint of the toes (Gibbons et al., 2002). Clinically, these lesions may produce long-standing, painful swelling. Most lesions are slowly progressive. Pathologically, PVNS is characterized by synovial inflammation, with giant cell proliferation, collagen, and lipid-laden macrophages. Treatment of PVNS is often surgical, with resection of the lesion. However a recurrence rate of $10 \%$ to $20 \%$ is reported in the focal form, and up to $50 \%$ in the diffuse form.

Rheumatoid nodules can be found in the feet of patients with long-standing inflammatory arthritides, most commonly rheumatoid arthritis. However, associated arthropathy is not a precondition for the development of a rheumatoid nodule. Rheumatoid nodules present clinically as painful soft tissue masses. Common locations in the foot include the heel fat pad and the toes.

Plantar fibromatosis is the most commonly encountered soft tissue neoplasm of the foot. This condition is characterized by focal deposition of cellular tissue and collagen, typically abutting the superficial aspect of the mid plantar fascia. Plantar fibromatosis is typically painless. Despite its benign nature, $75 \%$ of the lesions recur locally after surgical excision.

Malignant soft tissue tumors of the foot include malignant fibrous histiocytoma, liposarcoma, and synovial sarcoma, among others. They often demonstrate more heterogeneous signal, and are deeper in location and larger than benign soft tissue masses. However, differentiation between benign and malignant tumors based on MRI is in general nonspecific.

\section{Stress injury and stress fractures}

Stress fractures and insufficiency fractures are frequent in the midfoot and forefoot, and involve predominantly the metatarsals, the tarsal navicular bone, the hallucal sesamoids, and, less frequently, the toe phalanges. Before a stress fracture takes place, a condition termed "stress injury" or "stress reaction" occurs. During this period, edema, hyperemia, and osteoclastic activity develops within the stressed area of the bone. In this stage, the patient may refer symptoms related to overuse without detectable findings on conventional radiographs. MRI is a reliable diagnostic method for detection of stress injury before a fracture line is seen (Weishaupt and Schweitzer, 2002). As the biomechanical stress persists, a fracture develops. Periosteal reaction can be detected on plain films shortly after as an area of periosteal elevation or reactive periostitis occurs at the site of fracture. However, some areas of the foot present a challenge due to anatomical overlap. These include the hallucal sesamoids and the tarsal navicular and cuboid bones. MRI can accurately detect radiographically occult fractures in these locations.

\section{Diabetic foot}

MRI has been shown to be highly sensitive for detection and staging of a number of musculoskeletal infection such as cellulitis, soft tissue abscesses, and osteomyelitis (Schweitzer and Morrison, 2004). Compared to bone scintigraphy, MRI has superior specificity and better spatial resolution, and the diagnosis can be obtained without delays.

Acute osteomyelitis demonstrates hypointensity of the bone marrow on $T_{1}$ weighted images and increased signal on $T_{2}$-weighted and STIR images. MRI is highly sensitive to the detection of periostitis and some reports indicate that it may be more sensitive than plain film radiography. All the above findings can be observed in acute osteomyelitis very early, probably within hours of the onset of the infection, whereas it may take few days to up to 2 weeks to be seen on plain films. Post-contrast imaging in patients with osteomyelitis is always indicated, as it helps to establish the presence of associated soft tissue abscess or enhancing sinus tract adjacent to the affected bone. Discrimination between neuroarthropathy and infection may be difficult with any imaging technique. Typically, neuroarthropathy exhibits the characteristic findings of bone fragmentation, dislocations, cortical and periosteal thickening, joint effusion, and soft tissue swelling.

\section{Critical Parameters and Troubleshooting}

Motion artifacts can be reduced by carefully placing and securing the foot joint within the extremity coil. Wedging sponges should be used in order to provide stability. If these measures fail, conscious sedation can be administered. If sedation is not possible, shortening of the protocol can be attempted by reducing the acquisition matrix. This will, however, decrease resolution.

Phase-encoding artifacts may arise from the medial and lateral plantar neurovascular bundle. On transverse images, the flow-related artifact could obscure tendinous or ligamentous structures. In such cases, change of 

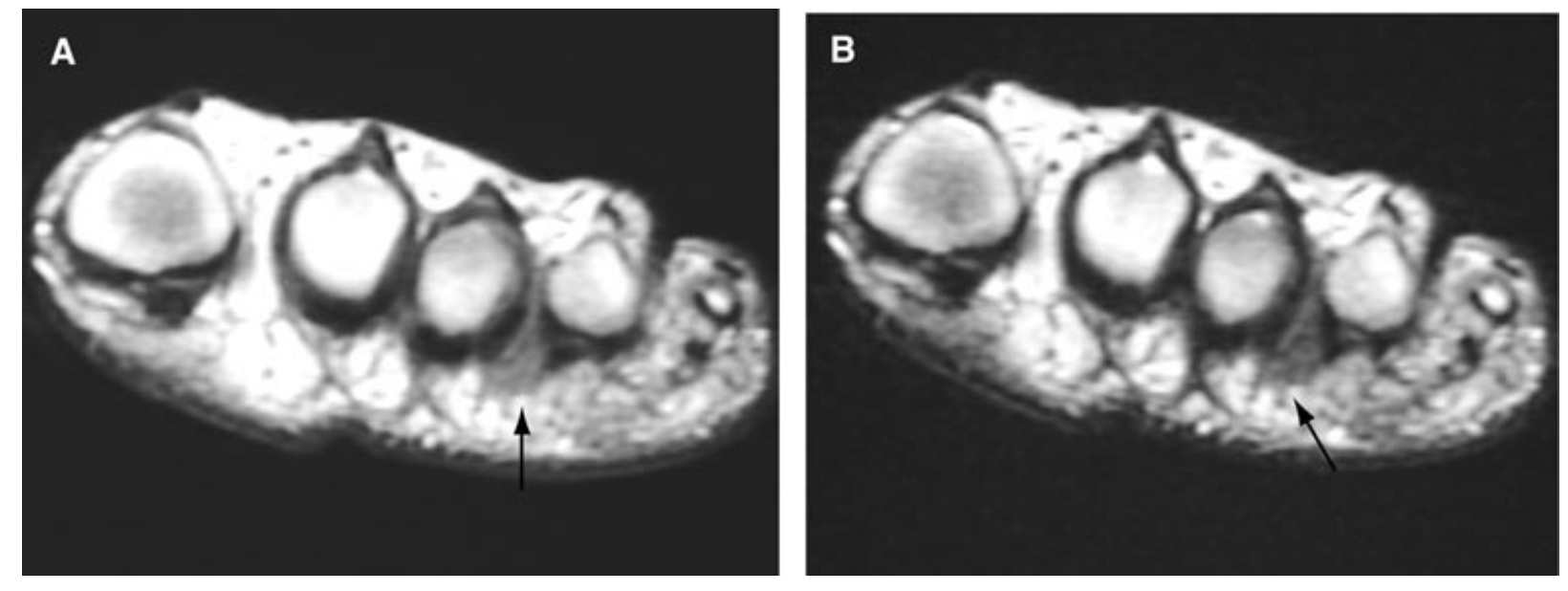

Figure A25.2.4 Morton's neuroma. (A) A coronal FSE proton density-weighted image demonstrates a well defined, teardrop-shaped mass emanating plantar to the third intermetatarsal ligament (arrow), consistent with Morton's neuroma. (B) A coronal FSE $T_{2}$-weighted image shows diffuse hypointensity within the mass (arrow) in keeping with fibrous composition.

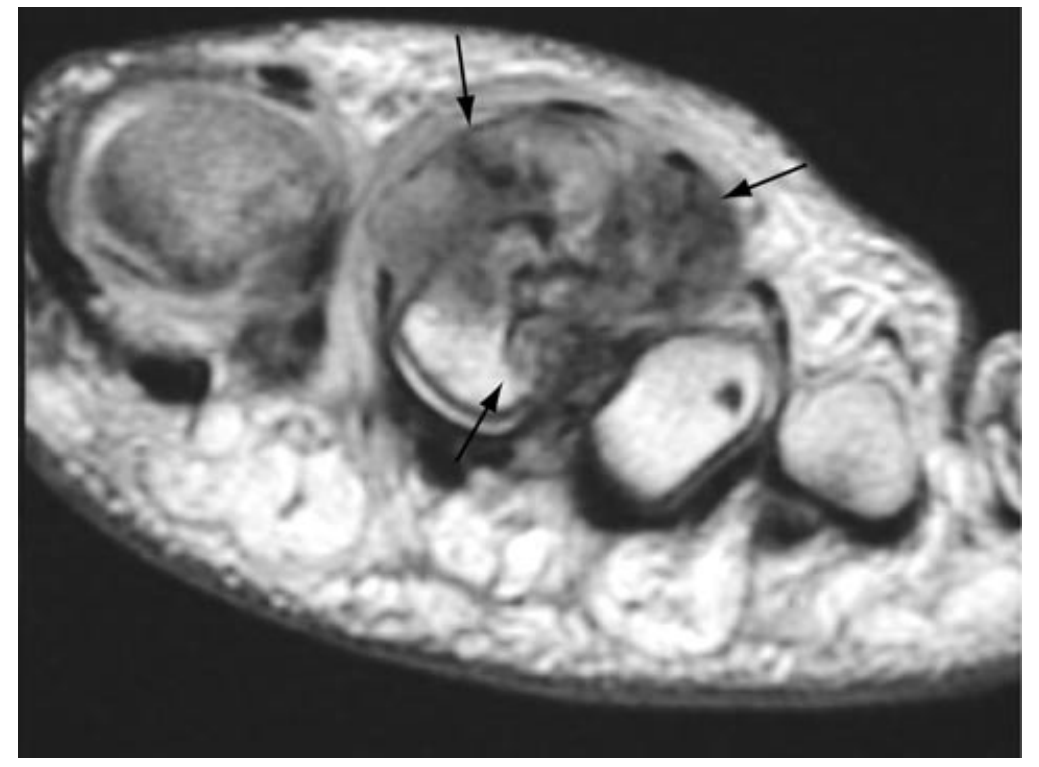

Figure A25.2.5 Pigmented villonodular synovitis. A coronal FSE proton density-weighted image shows a nodular heterogeneous mass eroding the second metatarsal head with multiple internal hypointense foci related to hemosiderin deposits (arrows).

the phase-encoding direction from anteriorposterior to right-to-left usually solves this problem.

\section{Anticipated Results}

\section{Morton's neuroma}

The behavior of Morton's neuroma on MRI imaging is fairly typical. The lesion demonstrates low to intermediate signal intensity on $T_{1}$ - and $T_{2}$-weighted spin-echo sequences due to its predominantly fibrous composition (Bencardino et al., 2000). Other soft-tissue masses may exhibit a similar signal pattern related to either low cellularity, high collagen content, or hemosiderin deposits, including those associated with fibromatosis, nodular fasciitis, and pigmented villonodular synovitis. However, hypointensity on $T_{1^{-}}$and $T_{2}$-weighted imaging combined to the typical location and distinct clinical history is characteristic of Morton's neuroma (Fig. A25.2.4).
Ankle and Foot

A25.2.11 
Patient position

Scan type

Imaging plane (orientation)

Central slice or volume center

Echo time $\left(T_{\mathrm{E}}\right)$

Repeat time $\left(T_{\mathrm{R}}\right)$

Flip angle (FA)

Fields of view $\left(\mathrm{FOV}_{x}, \mathrm{FOV}_{y}\right)$

Resolution $(\Delta x, \Delta y)$

Number of data points collected $\left(N_{x}, N_{y}\right)$

Slice thickness $(\Delta z)$

Number of slices

Slice gap

Number of acquisitions $\left(N_{\text {acq }}\right)$

Read direction

Scan time
Supine

2-D gradient echo

Oblique coronal

Position for whole foot coverage

$15 \mathrm{msec}$

$500 \mathrm{msec}$

$15^{\circ}$

$150 \mathrm{~mm}, 150 \mathrm{~mm}$

$0.2029 \mathrm{~mm}, 0.4567 \mathrm{~mm}$

512,224

$3 \mathrm{~mm}$

25

$00 \mathrm{~mm}$ (interleave)

2

Cranial-caudal

$7 \mathrm{~min}, 36 \mathrm{sec}$
MR Imaging of the Midfoot and Forefoot

A25.2.12

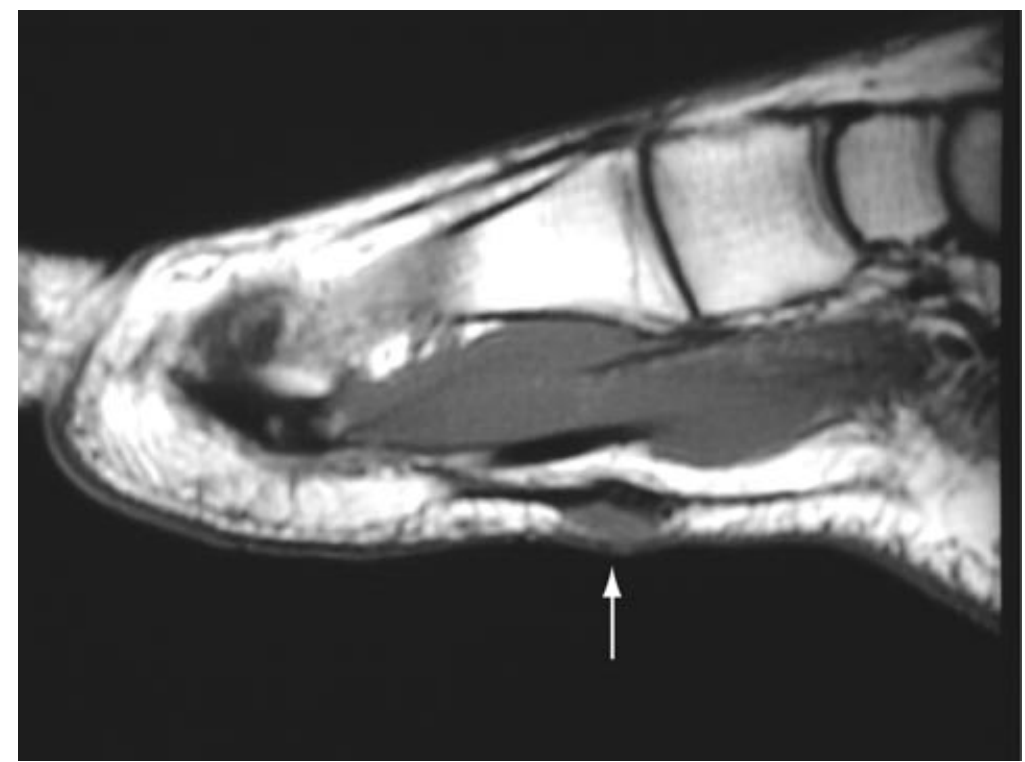

Figure A25.2.6 Plantar fibroma. A coronal FSE proton density-weighted image depicts a nodular mass isointense to muscle arising from the medial plantar aponeurosis (arrow). The mass has well defined borders inferiorly blending imperceptibly with the plantar fascia along its superior margin.

Some authors have advocated the use of shorttau inversion recovery (STIR) and gadoliniumenhanced images in order to increase the lesion's conspicuity (Unger et al., 1992; Terk et al., 1993). We believe that the diagnosis of Morton's neuroma in symptomatic patients could be performed based only on the information from standard $T_{1}$ - and $T_{2}$-weighted fast spin-echo images (Bencardino et al., 2000).

\section{Soft tissue masses}

MR imaging is being used extensively for evaluation of painful conditions of the ankle and foot, and it is not unusual to find gan- glion cysts when imaging a patient for nonspecific pain. The MR imaging manifestations of ganglion cysts are common in all locations, presenting as hypointense masses on $T_{1}$ weighted images and hyperintense masses on $T_{2}$-weighted images. Normally, they are rather well demarcated by a fibrous capsule and their "neck," or connection into an adjacent joint or tendon sheath, if present, can usually be well demonstrated with MRI due to the multiplanar capabilities of this technique. Lack of enhancement following intravenous gadolinium aids in distinguishing ganglions from solid masses. 


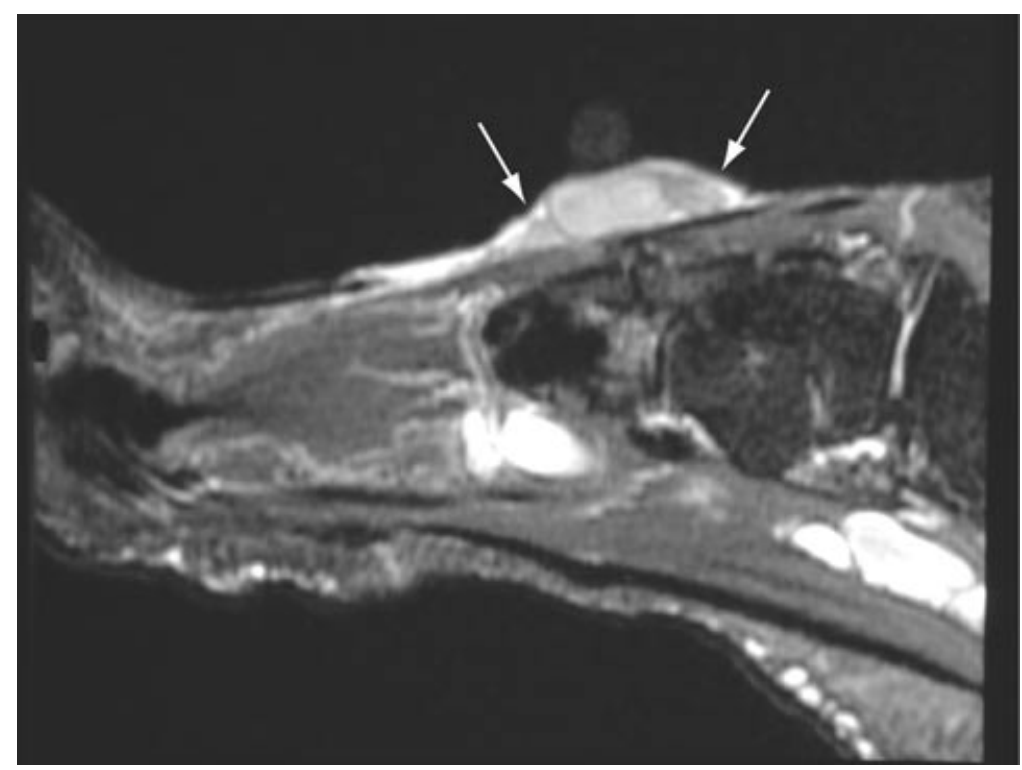

Figure A25.2.7 Malignant fibrous histiocytoma (MFH). A sagittal STIR image demonstrates a subcutaneous, heterogeneous mass tightly adherent to the dorsal aponeurosis (arrows). Despite its rather nonaggressive features on MRI, the lesion was histologically confirmed as MFH.

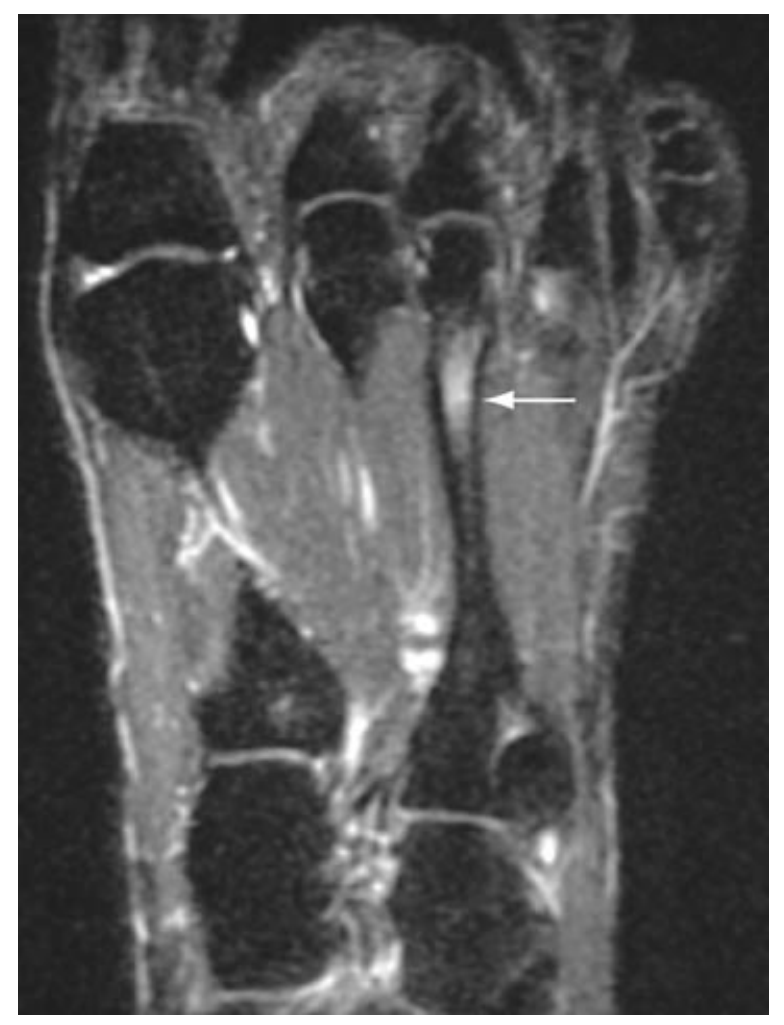

Figure A25.2.8 Stress reaction. A transverse fat-suppressed FSE proton density-weighted image shows a diffuse bone marrow edema involving the third metatarsal shaft in a runner (arrow). No discrete fractures are yet seen. 

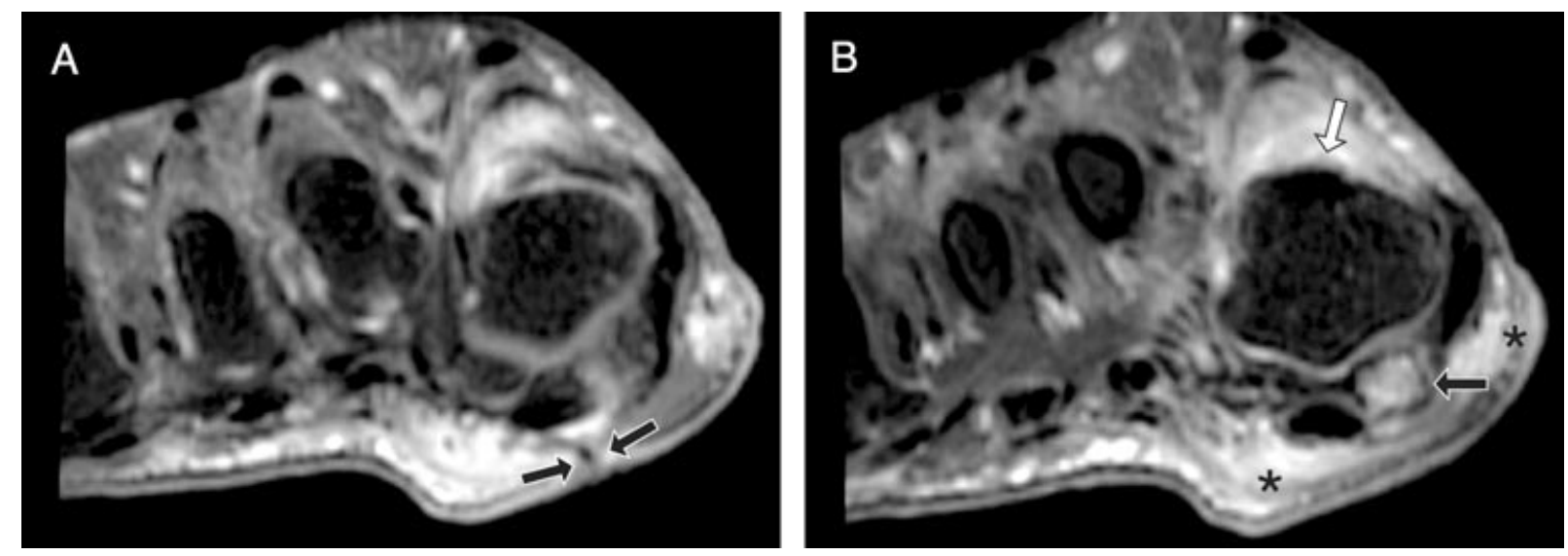

Figure A25.2.9 Osteomyelitis. (A) A coronal post-contrast fat-suppressed $T_{1}$-weighted image demonstrates a plantar ulcer to the first metatarsal phalangeal (MTP) joint. Discrete peripheral linear enhancement is consistent with sinus tract (arrows) extending into the hallucal sesamoid. (B) A coronal post-contrast fat-suppressed $T_{1}$-weighted image proximal to panel (A) shows enhancement of the hallucal sesamoid (filled arrow) associated with infectious synovitis (open arrow) of the first MTP joint capsule and cellulitis of the plantar fat pad (asterisks).

The MR imaging manifestations of PVNS are characteristic due to the paramagnetic effect of hemosiderin, producing focal areas of hypointensity on practically any pulse sequence, mixed with areas of hypointensity on $T_{1}$-weighted images and of hyperintensity on $T_{2}$-weighted or proton density-weighted images (Fig. A25.2.5; Jelinek et al., 1989; Lin et al., 1999). The short $T_{2}$ relaxation times produced by the presence of hemosiderin in the tissues is observed better using highfield magnets. Magnetic susceptibility artifact from hemosiderin is particularly pronounced on gradient echo pulse sequences (Table A25.2.10).

On MRI, rheumatoid nodules have a heterogeneous appearance with irregular margins probably reflecting surrounding fibrosis and focal areas of hyperintensity on $T_{2}$-weighted images (Sanders et al., 1998). Intense heterogeneous enhancement can be seen on postcontrast images.

Plantar fibromatosis is usually heterogeneous on all pulse sequences. Single or multiple hypointense nodules are typically noted within the central or medial plantar aponeurosis (Fig. A25.2.6). Hyperintense signal is often elicited with STIR imaging. Post-contrast enhancement varies on the amount of cellular components in each lesion.

MRI features that suggest malignancy include signal heterogeneity with extensive peritendinous growth and infiltrative behavior with intermediate signal intensity on $T_{2}$-weighted images (Fig. A25.2.7; Waldt et al., 2003).

\section{Stress injury and stress fractures}

Stress injury is manifested on MR imaging as poorly defined abnormal signal intensity of the bone marrow, similar to a bone contusion, with an ill-defined area of hypointensity on $T_{1}$-weighted images and hyperintensity on $T_{2}$ weighted images and fat-suppressed pulse sequences (Fig. A25.2.8; Sijbrandij et al., 2002). As the stress persists and a fracture develops, MRI will show a hypointense irregular line within the area of edema and hyperemia. Periosteal callous formation begins shortly after the fracture, and it can be seen on MRI as a hypointense line parallel to the cortex, representing the elevated periosteum. The periosteum is separated from the underlying cortex by hyperintense tissue on $T_{2}$-weighted images, most likely representing inflammatory reaction.

MRI has been found to be more sensitive than conventional radiography and more specific than bone scintigraphy for detecting occult fractures, particularly in the elderly and in osteoporotic patients (Deutsch et al., 1989). Acute post-traumatic fractures will have similar MRI manifestations than stress-related fractures, with hypointense lines representing the fracture, surrounded by ill-defined areas of edema and hemorrhage.

\section{Diabetic foot}

Depiction of bone marrow signal abnormality manifested as low signal intensity on $T_{1}$-weighted images and hyperintensity on $T_{2}-$ weighted images in continuity with a skin ulcer or adjacent subcutaneous abscess are
MR Imaging of the Midfoot and Forefoot

A25.2.14 
indicative of osteomyelitis. Distinguishing features with neuroarthropathy include bone marrow changes in the absence of adjacent soft tissue infection. Gadolinium-enhanced MR imaging using fat-suppressed $T_{1}$-weighted images in the sagittal and coronal oblique planes may provide additional information regarding the extent of soft tissue abnormality as well as depiction of sinus tracts and abscesses. See Figure A25.2.9.

\section{Literature Cited}

Ashman, C.J., Klecker, R.J., and Yu, J.S. 2001. Forefoot pain involving the metatarsal region: Differential diagnosis with MRI imaging. $R a$ diographics 21:1425-1440.

Bencardino, J., Rosenberg, Z.S., Beltran, J., Liu, X., and Marty-Delfaut, E. 2000. Morton's neuroma: Is it always symptomatic? Am. J. Roentgenol. 175:649-653.

Deutsch, A.L., Mink, J.H., and Waxman, A.D. 1989. Occult fractures of the proximal femur: MR imaging. Radiology 170:113-116.

Gibbons, C.L., Rhwaja, H.A., Cole, A.S., Cooke, P.H., and Athanasou, N.A. 2002. Giant cell tumour of the tendon sheath in the foot and ankle. J. Bone Joint Surg. Br. 84:1000-1003.

Jelinek, J.S., Kransdorf, M.J., Utz, J.A., Berrey, B.H. Jr., Thomson, J.D., Heekin, R.D., and Radowich, M.S. 1989. Imaging of pigmented villonodular synovitis with emphasis on MR imaging. Am. J. Roentgenol. 152:337-342.

Lin, J., Jacobson, J.A., Jamadar, D.A., and Ellis, J.H. 1999. Pigmented villonodular synovitis and related lesions: The spectrum of imaging findings. Am. J. Roentgenol. 172:191-197.

Rubin, D.A., Towers, J.D., and Britton, C.A. 1996. MR imaging of the foot: Utility of complex oblique imaging planes. Am. J. Roentgenol. 166:1079-1084.

Sanders, T.G., Linares, R., and Su, A. 1998. Rheumatoid nodule of the foot: MRI appearances mimicking an indeterminate soft tissue mass. Skeletal Radiol. 27:457-460.

Schweitzer, M.E. and Morrison, W.B. 2004. MR imaging of the diabetic foot. Radiol. Clin. North Am. 42:61-71.
Shellock, F.G. 2007. Reference Manual for Magnetic Resonance Safety, Implants and Devices, 2007 Edition. Biomedical Research Publishing Group, Los Angeles, Calif.

Sijbrandij, E.S., van Gils, A.P., and de Lange, E.E. 2002. Overuse and sports-related injuries of the ankle and hindfoot: MR imaging findings. Eur. J. Radiol. 43:45-56.

Terk, M.R., Kwong, P.K., Suthar, M., Horvath, B.C., and Colleti, P.M. 1993. Morton neuroma: Evaluation with MR imaging performed with contrast enhancement and fat suppression. Radiology 189:239-241.

Unger, H.R. Jr, Mattoso, P.Q., Drusen, M.J., and Neumann, C.H. 1992. Gadopentate-enhanced magnetic resonance imaging with fat saturation in the evaluation of Morton's neuroma. J. Foot Surg. 31:244-246.

Waldt, S., Rechl, H., Rummeny, E.J., and Woertler, K. 2003. Imaging of benign and malignant soft tissue masses of the foot. Eur. Radiol. 13:11251136.

Weishaupt, D. and Schweitzer, M.E. 2002. MR imaging of the foot and ankle: Patterns of bone marrow signal abnormalities. Eur. Radiol. 12:416-426.

Weishaupt, D., Schweitzer, M.E., Morrison, W.B., Haims, A.H., Wapner, K., and Kahn, M. 2001. MRI of the foot and ankle: Prevalence and distribution of occult and palpable ganglia. J. Magn. Reson. Imaging 14:464-471.

Weisthaupt, D., Treiber, K., Kundert, H.P., Zollinger, H., Vienne, P., Hodler, J., Willman, J.K., Marincek, B., and Zanetti, M. 2003. Morton neuroma: MR imaging in prone, supine and upright weight bearing body positions. Radiology 226:849-856.

Zlatkin, M.B. 1999. Techniques for MR imaging of joints in sports medicine. Magn. Reson. Imaging Clin. N. Am. 7:1-21.

Contributed by Jenny Bencardino and Zehava S. Rosenberg

Hospital for Joint Diseases

NYU Medical Center

New York, New York
Ankle and Foot

A25.2.15 